\title{
Preoperative ultrasound mapping of the vagus nerve in thyroid surgery
}

\author{
Sophie Bidault ${ }^{1}$, Elizabeth Girard ${ }^{1}$, Marie Attard ${ }^{1}$, Gabriel Garcia ${ }^{1}$, Joanne Guerlain ${ }^{2}$, Ingrid Breuskin ${ }^{2}$, \\ Eric Baudin ${ }^{3}$, Julien Hadoux ${ }^{3}$, Camilo Garcia ${ }^{3}$, Livia Lamartina ${ }^{3}$, Dana M. Hartl ${ }^{2} \wedge$ \\ ${ }^{1}$ Department of Radiology, Gustave Roussy Cancer Campus and University Paris-Saclay, Gustave Roussy, Villejuif, France; ${ }^{2}$ Department of Surgery, \\ Anesthesia and Interventional Medicine, Thyroid Surgery Unit, Gustave Roussy, Villejuif, France; ${ }^{3}$ Department of Nuclear Medicine and Endocrine \\ Oncology, Gustave Roussy Cancer Campus and University Paris-Saclay, Gustave Roussy, Villejuif, France \\ Contributions: (I) Conception and design: S Bidault, DM Hartl; (II) Administrative support: E Baudin; (III) Provision of study materials or patients: \\ All authors; (IV) Collection and assembly of data: S Bidault, DM Hartl, E Girard, J Guerlain, I Breuskin, M Attard, G Garcia; (V) Data analysis and \\ interpretation: S Bidault, DM Hartl; (VI) Manuscript writing: All authors; (VII) Final approval of manuscript: All authors. \\ Correspondence to: Dana M. Hartl. Department of Surgery, Anesthesia and Interventional Medicine; Thyroid Surgery Unit, Gustave Roussy Cancer \\ Campus and University Paris-Saclay, Gustave Roussy, 114 rue Edouard Vaillant, 94805 Villejuif, Cedex, France. Email: dana.hartl@gustaveroussy.fr.
}

\begin{abstract}
Backgroundk Intraoperative neuromonitoring (IONM) in thyroid surgery requires electric stimulation of the vagus nerve to verify correct electrode placement. Classically the nerve is found deep to or in-between the common carotid artery and internal jugular vein, but previous studies have shown that the nerve can sometimes be found superficial to the vessels. Our aim was to determine the incidence of a superficial vagus nerve using ultrasound (US) and study possible clinical factors associated with an anteriorly-located vagus nerve.
\end{abstract}

Methods: Retrospective study of patients undergoing thyroid surgery (lobectomy or total thyroidectomy) with intermittent IONM. Substernal goiters, locally invasive tumors or bulky lymph nodes were excluded. The vagus nerve was identified at the level of the mid-thyroid lobe on each side on preoperative US performed by two specialized radiologists, and its location according to 6 possible positions in relationship to the common carotid artery was recorded. The anatomic variability of the vagus nerve was analyzed in relationship to patient demographics and thyroid pathology.

Results: Five-hundred twenty-seven patients were included. The right vagus nerve ( $\mathrm{n}=522)$ was in-between, superficial or deep to the vessels in $92.3 \%, 6.1 \%$ and $1.5 \%$ and of cases, respectively, and the left vagus ( $n=517)$ in $80.2 \%, 18.6 \%$ and $1.2 \%$ of cases, respectively, with a statistically significant difference between right and left vagus nerves $(\mathrm{P}<0.001)$. The type of pathology, size of the dominant nodule or the volume of the thyroid lobe were not correlated to finding a superficial vagus nerve.

Conclusions: The vagus nerve was identified in all cases on US and found to be anterior to common carotid artery at the level of the thyroid lobe in $18.6 \%$ of cases on the left and $6.1 \%$ of cases on the right. Identifying this anatomic variant preoperatively may facilitate IONM and avoid inadvertent trauma to the vagus nerve during thyroid surgery.

Keywords: Vagus nerve; recurrent nerve; intraoperative nerve monitoring (IONM); thyroid surgery

Submitted Aug 24, 2021. Accepted for publication Nov 05, 2021.

doi: $10.21037 /$ gs-21-580

View this article at: https://dx.doi.org/10.21037/gs-21-580

$\wedge$ ORCID: 0000-0003-1161-5628. 


\section{Introduction}

Recurrent laryngeal nerve injury is a possible complication of thyroid surgery. Permanent paralysis of the recurrent nerve is rare in experienced hands but can significantly impair patients' quality of life. Intraoperative neuromonitoring (IONM) in thyroid surgery is frequently employed as a means to map and test the recurrent nerve and to reduce the rate of recurrent nerve paralysis in some instances $(1,2)$. Localization and stimulation of the vagus nerve is recommended as the first step for accurate IONM. Previous knowledge of the anatomy of the vagus nerve has been based on cadaver studies, with few intraoperative surgical studies of the location of the vagus nerve during thyroidectomy (3). Today, using standard commercially available ultrasound (US) equipment, the vagus nerve can be easily identified and mapped in vivo $(4,5)$. Ultrasound has been used to evaluate the vagus nerve in different neurological diseases such as Parkinson's disease and inflammatory polyneuropathy (6-8), and to map the vagus nerve before transcutaneous thermoablation techniques for thyroid nodules (4).

Our previous study showed that the US location of the vagus nerve and intraoperative findings were comparable in $95 \%$ of cases (5). This previous study of 82 patients also found that the vagus nerve, instead of being deep to the great vessels at the level of the thyroid lobes, was in a superficial position, anterior to the common carotid artery in $24.4 \%$ of cases on the left and in $2.4 \%$ of cases on the right. The aim of the present study was to verify these findings in a larger cohort and to identify, if possible, factors associated with this anatomic variation.

We present the following article in accordance with the STROBE reporting checklist (available at https:// gs.amegroups.com/article/view/10.21037/gs-21-580/rc).

\section{Methods}

This was a retrospective study of consecutive patients undergoing routine US before thyroid lobectomy or total thyroidectomy with or without central neck dissection or before reoperative surgery for thyroid cancer. Patients with previous external beam radiation therapy to the neck or preoperative laryngeal immobility were excluded, as were patients with substernal goiters, locally invasive cancers or bulky metastatic nodes in the central and/or lateral neck compartments as these factors could impair the visualization of the vagus nerve on US or modify its position. The location of the vagus nerve was assessed systematically with dynamic US images during preoperative US examination. Preoperative US is routine standard of care for all patients at our institution (9) and the location of the vagus nerve is routinely recorded in a schematic representation in the patients' files since January 2017. The study was conducted in accordance with the Declaration of Helsinki (as revised in 2013). The study was approved by the institutional ethics board at Gustave Roussy and individual consent for this retrospective analysis was waived.

US was performed with the Toshiba/Canon Aplio 500 and Aplio i900 ultrasound devices using linear probes at 10-18 Mhz en B mode and Doppler (Canon Medical Systems). The location of the vagus nerve on a horizontal axis at approximately the midlevel of the thyroid lobe (or approximately $2 \mathrm{~cm}$ below the lower border of the cricoid cartilage) was recorded according to 6 positions (Figure 1): positions 1 and 2 were considered as "in-between" the vessels, positions 3 and 4 were considered as "superficial", and positions 5 and 6 as "deep" to the great vessels. Patient demographics, benign versus malignant pathology, a primary versus a reoperative setting, nodule size and thyroid lobe volume were analyzed from computerized patient files.

\section{Statistical analysis}

Statistical analysis with descriptive statistics and nonparametric analysis was performed using the SPSS software (version 26.0.0.1, 2019 SPSS Inc., Chicago, IL, USA).

\section{Results}

Five hundred twenty-seven patients were included, with identification of 522 right vagus nerves and 517 left vagus nerves (for a total of 1,039 nerves). In some cases, before thyroid lobectomy, only one vagus nerve was evaluated with US, but both nerves were evaluated in the same patient in 512 patients. These patients included the 82 patients evaluated in our previous study (5). Table 1 shows demographics and thyroid pathology.

Table 2 shows the US location of the vagus nerve relative to the great vessels. The right vagus nerve $(\mathrm{n}=522)$ was inbetween, superficial or deep or to the vessels in $92.3 \%$, $6.1 \%$ and $1.5 \%$ and of cases, respectively, and the left vagus $(\mathrm{n}=517)$ in $80.2 \%, 18.6 \%$ and $1.2 \%$ of cases, respectively, with a statistically significant difference between right and left vagus nerves. On the left there was a higher rate of 


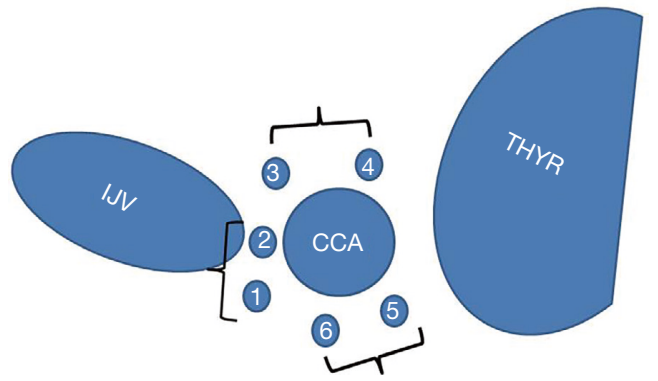

Figure 1 Schematic representation of the location of the vagus nerve on the right side: locations 1 and 2 were "between" the great vessels, locations 3 and 4 were considered "superficial," or "anterior" and locations 5 and 6 were considered as "deep" to the vessels. THYR, thyroid lobe; IJV, internal jugular vein; CCA, common carotid artery.

Table 1 Demographics of the cohort

\begin{tabular}{|c|c|}
\hline Demographics & Values \\
\hline $\mathrm{N}$ (female/male) & $527(393 / 134)$ \\
\hline Average age, years ( \pm standard deviation) & $48( \pm 16)$ \\
\hline Min-Max & $8-87$ \\
\hline Median age, years & 48 \\
\hline Nerves identified (right/left) & $522 / 517$ \\
\hline Cancer & $338(64.1 \%)$ \\
\hline Previous surgery (no/yes) & $451(85.6 \%) / 76(14.4 \%)$ \\
\hline Nodule side (right/left) & $46 \% / 54 \%$ \\
\hline \multicolumn{2}{|l|}{ Dominant nodule or cancer size } \\
\hline Average & $28 \mathrm{~mm}$ \\
\hline Standard deviation & $\pm 17 \mathrm{~mm}$ \\
\hline Min-Max & $2-107 \mathrm{~mm}$ \\
\hline \multicolumn{2}{|l|}{ Right lobe volume } \\
\hline Average & $15 \mathrm{~cm}^{3}$ \\
\hline Standard deviation & \pm 18 \\
\hline Min-Max & $1-146 \mathrm{~cm}^{3}$ \\
\hline \multicolumn{2}{|l|}{ Left lobe volume } \\
\hline Average & $17 \mathrm{~cm}^{3}$ \\
\hline Standard deviation & \pm 23 \\
\hline Min-Max & $1-215 \mathrm{~cm}^{3}$ \\
\hline
\end{tabular}

Table 2 US localization of the vagus nerve as compared to the internal jugular vein and the common carotid artery at the midthyroid-lobe level (approximately $2 \mathrm{~cm}$ below the inferior border of the cricoid cartilage)

\begin{tabular}{lcc}
\hline Vagus nerve location & Right vagus $(\mathrm{n}=522)$ & Left vagus $(\mathrm{n}=517)$ \\
\hline Between the vessels & $482(92.3 \%)$ & $415(80.2 \%)$ \\
Position 1 & $411(78.7 \%)$ & $315(60.9 \%)$ \\
Position 2 & $71(13.6 \%)$ & $100(19.3 \%)$ \\
Superficial to vessels & $32(6.1 \%)$ & $96(18.6 \%)$ \\
Position 4 & $13(2.5 \%)$ & $66(12.8 \%)$ \\
Position 3 & $19(3.6 \%)$ & $30(5.8 \%)$ \\
Deep to vessels & $8(1.5 \%)$ & $6(1.2 \%)$ \\
Position 5 & 0 & $1(0.2 \%)$ \\
Position 6 & $8(1.5 \%)$ & $5(1 \%)$ \\
\hline
\end{tabular}

anteriorly-located vagus nerves: $18.6 \%$ versus $6.1 \%$ on the right, which was statistically significant (Fisher's exact test, two-sided $\mathrm{P}$ value $<0.001)$. The vagus nerve was anterior to the common carotid artery on both sides in $16 / 512$ patients in whom both nerves were evaluated. Right-left symmetry of the vagus nerve in the same patient ( $\mathrm{n}=512$ patients) was observed in $80.7 \%(413 / 512)$, with asymmetry in $19.3 \%$ (99/512). Left-right asymmetry was not statistically related to the nodule size or to the diagnosis of cancer. Figures 2-7 give examples of US images of the vagus nerve and its relationship to the common carotid artery and internal jugular vein (Figures 2-7).

Of the total 527 patients, 451 (85.6\%) underwent primary surgery and $76(14.4 \%)$ were reoperative cases. For the position of the right vagus nerve, there was no difference between primary and reoperative patients: the rate of a superficial right vagus nerve was $6 \%$ in primary surgery patients and $6.6 \%$ for reoperative patients $(\mathrm{P}=0.25)$. For the left vagus nerve, a superficial location was observed in $18.8 \%$ of primary surgery patients versus $14.5 \%$ of reoperative surgery patients $(\mathrm{P}=0.07)$. Age, gender, nodule pathology, and nodule size did not statistically influence the position of the vagus nerve on the left or on the right $(\mathrm{P}>0.5$ in all instances). The volume of the right lobe did not influence the position of the vagus nerve on the right or the rate of an anteriorly-located vagus nerve on the right $(\mathrm{P}=0.19$ 


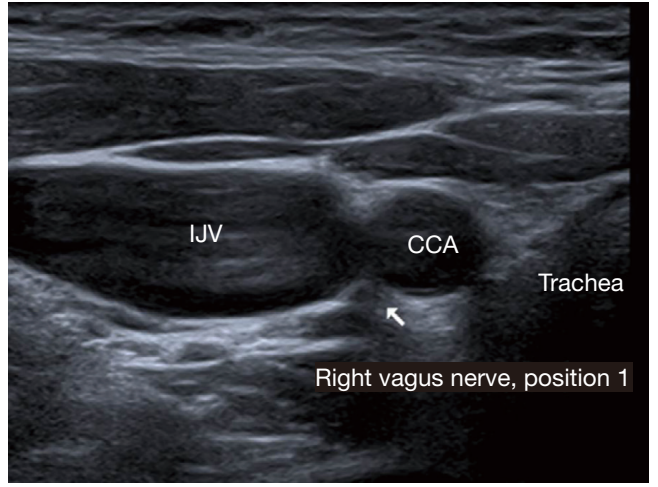

Figure 2 Ultrasound aspect of the right vagus nerve (white arrow) in position 1. THYR, thyroid lobe; IJV, internal jugular vein; CCA, common carotid artery.

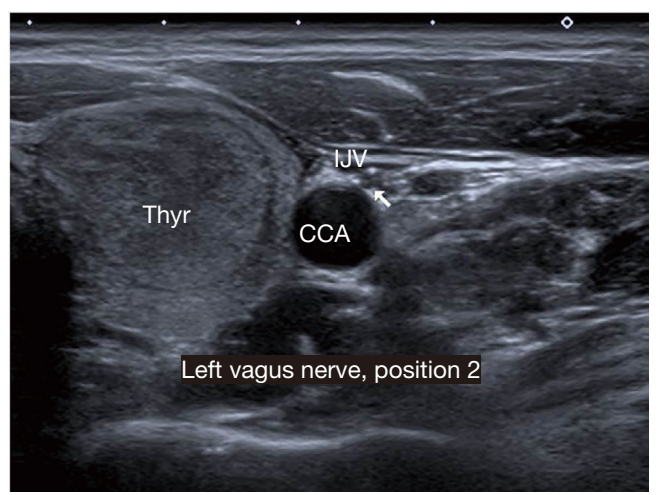

Figure 3 Ultrasound aspect of the left vagus nerve (white arrow) in position 2. THYR, thyroid lobe; IJV, internal jugular vein; CCA, common carotid artery.

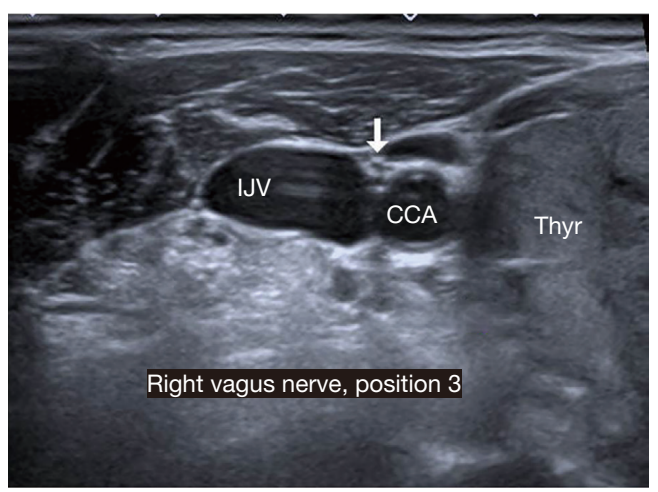

Figure 4 Ultrasound aspect of the right vagus nerve (white arrow) in position 3. THYR, thyroid lobe; IJV, internal jugular vein; CCA, common carotid artery.

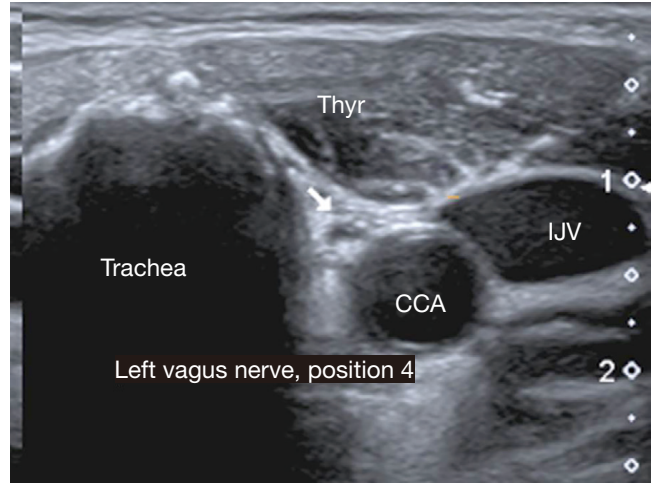

Figure 5 Ultrasound aspect of the left vagus nerve (white arrow) in position 4. THYR, thyroid lobe; IJV, internal jugular vein; CCA, common carotid artery.

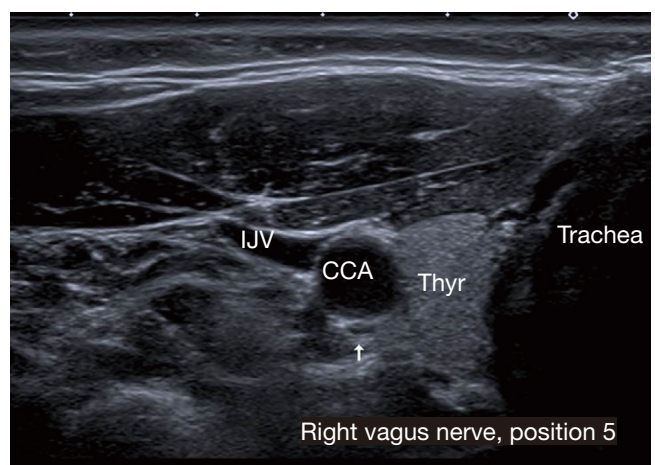

Figure 6 Ultrasound aspect of the right vagus nerve (white arrow) in position 5. THYR, thyroid lobe; IJV, internal jugular vein; CCA, common carotid artery.

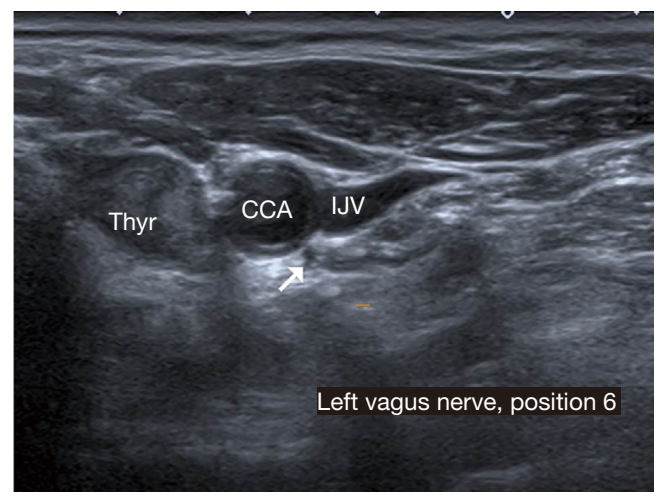

Figure 7 Ultrasound aspect of the left vagus nerve (white arrow) in position 6. THYR, thyroid lobe; IJV, internal jugular vein; CCA, common carotid artery. 
and $\mathrm{P}=0.3$, respectively). However, on the left, an anterior vagus nerve was significantly related to a lower lobe volume. The lobe volume for cases with an anteriorly-located vagus nerve was $13 \pm 19$ versus $18 \pm 24 \mathrm{~cm}^{3}$ for cases with a vagus nerve deep to or in-between the great vessels $(\mathrm{P}=0.036)$.

\section{Discussion}

The term "vagus" means "wandering". The vagus, or the tenths cranial nerve, is the longest cranial nerve, coursing from the nucleus ambiguous in the brainstem through the jugular foramen then to the neck and thorax all the way to the abdomen (10). It carries both somatic and visceral afferents and efferents with motor, sensory and autonomous parasympathetic nerve fibers. Its complex functions include regulation of breathing, digestion, heart rate and blood pressure, inflammatory response and symptoms of stress or anxiety, as well as motor functions ensuring pharyngeal and vocal fold mobility, in particular.

In a report dating back to 1915 , A. Gibson cites several anatomical studies from the $19^{\text {th }}$ century describing an "abnormal relationship" of the cervical vagus in its relationship to the common carotid artery (11). Gibson described a rare finding in a 59-year-old male subject of both vagus nerves running anteriorly to the common carotid arteries. According to his literature search at that time, only three cases of an anteriorly-located vagus nerve had been described in the literature on the right side, whereas a much larger proportion of anteriorly-located vagus nerves on the left had been observed. In one cited study of 50 cases, "the majority" of the left vagus nerves were anterior to the common carotid artery starting from the level of the upper border of the thyroid cartilage all the way down to the thoracic inlet. In a second study, 11/30 cases had a left anterior vagus nerve. The theory of the day was that the anterior location was related to age. The 8 fetal and infant subjects in a study published by Casali in 1911 and cited by Gibson all had a left vagus nerve coursing anteriorly to the common carotid artery. Of the 24 fetuses studied by Gibson himself, however, none had an anterior vagus nerve on either side. Today, there is still no satisfactory explanation for this "abnormal" anterior location of the vagus nerve. Some have hypothesized that the relationship between the vagus nerve and the cervical plexus may alter the location of the vagus nerve, but the most plausible explanation would seem to be embryological due to variations in the fusion of the carotid sheath with other fascial planes during development. This does not explain, however, the left-sided predominance of this phenomenon $(5,12)$.

Today's US technology allows precise visualization of peripheral nerves. The diameter of the vagus nerve in the neck has been widely studied in healthy adults and children and in various diseases (13). The vagus nerve on the right in the mid-neck has been shown to have a larger diameter than the left vagus nerve $(14,15)$. US measurements of the diameter of the vagus nerve have been studied in Parkinson's disease $(6,16)$, bulbar atrophy $(17)$, inflammatory polyneuropathies $(7,18)$, and neurosarcoidosis (19). US guidance has been largely employed for neurostimulator placement in epilepsy $(10,20)$ and has also recently been employed for microelectrode insertion into the vagus nerve for electrophysiologic microneurography (21).

The trajectory and anatomic relationships of the vagus nerve, particularly pertaining to IONM for thyroid surgery, have not been widely studied. Identification of the vagus nerve is necessary for intra-operative neuromonitoring during thyroid surgery. Dissection of the carotid sheath may put medially- or anteriorly-positioned vagus nerves at risk of inadvertent injury from dissection or from energy devices. Heat diffusion during radiofrequency ablation of thyroid nodules may also put an anteriorly- or mediallypositioned vagus at risk, and US localization of the vagus may be useful before thermoablation particularly of large nodules (4). Identification and dissection of the vagus nerve at the level of the thyroid lobes is also required for electrode placement for continuous intra-operative nerve monitoring (CIONM) (22-24). With this technique, the vagus nerve is electrically stimulated at regular intervals during the entire surgical procedure. A decrease in the amplitude and/or an increase in the latency of the electromyographic signal detected by the surface electrodes of the endotracheal tube on the vocal folds is an early sign of nerve trauma. This early "warning" during CIONM may enable the surgeon to avoid further, irreversible injury to the nerve by modifying the surgical maneuvers (25-27).

On US, the vagus nerve in the lower portion of the neck is recognized as a "thin band" in the posterior angle formed between the common carotid artery and the internal jugular vein (28). Already in 1998, Knappertz et al. used US to visualize the vagus nerve in 100 normal subjects, and then in 2001 Giovagnorio et al. used US to analyze the vagus nerve in 144 normal subjects $(28,29)$. Neither author reported a variation with regards to the classic, posterior position.

Chen et al. reported a study of 314 patients (628 vagus nerves) evaluated using US between 2015 and 2016 (30). The efficacy of visualization of the lower vagus nerve was 
$99.6 \%$ (626/628 nerves were seen). At the upper level of the neck in 551 cases, however, they found that $89.8 \%$ of the vagus nerves were in a lateral position as compared to the internal carotid artery, 3\% were located medially, 1.6\% were located anteriorly and $5.4 \%$ of nerves were posterior to the internal carotid artery. The left vagus nerve had significantly more anatomic variation in the upper neck than the right vagus which was more often lateral to the artery $(92.8 \%$ on the right versus $86.8 \%$ on the left).

In the study by Park et al. identifying the vagus nerve at the level of the thyroid using US, the anterior variation of the vagus nerve was observed in $4.3 \%$ of cases $(14 / 326)$ with a predominance on the left side (10 cases on the left, versus 4 cases on the right) (31). The medial variation was seen in only $1.2 \%$ of cases, 3 on the right and 1 on the left.

Dionigi et al. studied the surgical anatomy of the vagus nerve in the context of IONM identifying the vagus nerve using electric neurostimulation (3). In 140 consecutive patients (280 vagus nerves) they found that $96 \%$ of the vagus nerves were deep to the common carotid artery and internal jugular vein. They noted an "in-between and deep" position in $77 \%$ of vagus nerves on the right side and $73 \%$ on the left. In only $4 \%$ of cases were the vagus nerves superficial to the carotid-jugular axis and no apparent difference between the right and left sides for this configuration was noted. The difference in the rate of anteriorly-located vagus nerves between our study and that of Dionigi et al. may be related to the use of an electric stimulation to locate the nerve without performing an anatomic dissection. In the study by Dionigi et al. the nerve stimulator was set at $1-3 \mathrm{~mA}$ to electrically locate the nerve along the carotid sheath. If the nerve was not easily located at $1 \mathrm{~mA}$, the stimulation was increased to 2 or $3 \mathrm{~mA}$. At this level of stimulation, some diffusion of the electricity may be expected, so the nerve may be a few millimeters away and still be stimulated. This may have introduced some inaccuracy in the exact location of the vagus nerve in this electrical study.

Ha $e t$ al. defined 4 types of vagus nerves on US mapping: a usual type, in-between the great vessels; an anterior type, superficial to the common carotid artery; a medial type, medial to the common carotid artery; and a posterior type, behind the carotid artery (4). Out of 607 vagus nerves, they found that the in-between type occurred in $91.7 \%$ of cases on the right, with the anterior type occurring in $6.9 \%$ of cases and the other locations in only $1.3 \%$ of cases. On the left, however, they found that the in-between type occurred in only $62.2 \%$ of cases and that the anterior type occurred in $35.2 \%$ of cases. On the left, the other two types (medial and posterior) were only observed in $2.6 \%$ of cases.

Preoperative knowledge of the location of the vagus nerve can save precious time when stimulating the vagus nerve at the beginning of the thyroid operation (V1, in the guidelines) (1). Preoperative Us may enable the surgeon to avoid unnecessary dissection of the carotid sheath in the case of anteriorly-located nerves and limit the dissection between the carotid artery and the internal jugular vein if the vagus nerve lies medially to the carotid artery. Finding the vagus nerve for electrode placement for CIONM can be faster with the knowledge from preoperative US, as well. Knowing that there is an anteriorly-located vagus nerve may also warn the surgeon, avoiding inadvertent traction, coagulation or blind dissection in the region of the carotid sheath at the beginning of the operation.

Knowing where the vagus nerve is located may also be helpful in remote-access endoscopic and robot-assisted techniques such as the axillary, retroauricular, transoral or bilateral axillary breast approach (BABA). IONM in remote-access surgery seems to be more difficult and less reliable than for open surgery, and CIONM is not frequently reported in these remote-access techniques. A review by Dionigi et al. published in 2017 found only 9 studies reporting the use of IONM during robot-assisted or endoscopic thyroidectomy (32). Vagus nerve stimulation according to the IONM guidelines was performed in only $30 \%$ of the reported cases. For robot-assisted retroauricular (facelift) and transoral approaches, the standard procedure for IONM with stimulation of the vagus nerve as well as the recurrent nerve was only applied in $76 \%$ of facelift robotic cases and $17 \%$ of transoral cases in the study by Ji et al. (33). Knowing exactly where to dissect and stimulate the carotid sheath at the beginning of the operation or where to dissect to place the electrode for CIONM could facilitate IONM and possibly increase the use of the standardized, recommended procedure. In addition, in the axillary approach, in particular, the robotic arms are placed between the two heads of the sterno-mastoid muscle and may come into close contact with the carotid artery. Knowing that the vagus nerve is superficially located along the carotid sheath may make the surgeon more careful during the dissection and robotic arm placement.

The anteriorly located vagus nerve seems more vulnerable in open surgery due to the fact that the carotid sheath is approached from its anterior aspect through the neck incision. During thermal ablation of thyroid nodules with radiofrequency, laser, microwave or ethanol ablation, the heat (or ethanol) applied to the nodule may in rare instances 
go beyond the thyroid capsule and endanger other anatomic structures (34). In these cases, a medially-positioned vagus nerve may be more vulnerable to injury (4). Knowledge of the location of the vagus nerve can be very valuable to physicians performing these techniques, to inform patients and to attempt to avoid spread of heat or ethanol in the presence of a vulnerable nerve.

Our previous study and this current study more closely resemble that of Ha et al. In our previous study, the correlation between US localization of the vagus nerve and in-vivo surgical anatomy was exact in $96 \%$ cases for the right vagus nerves and in $95 \%$ of cases for the left vagus nerves (5). Most interestingly, we found that on US, the anterior position on the left occurred in $24.4 \%$ of cases while on the right it was seen in $2.4 \%$ of cases. This tendency has been confirmed in the present study with $18.6 \%$ of anteriorly-located vagus nerves on the left and $6.1 \%$ on the right. The deep variant was seen on US in our first study in $3.6 \%$ of cases on the left and on the right, but in the present study this variant was seen in $1.5 \%$ of cases on the right and $1.2 \%$ of cases on the left.

The discrepancy between our 2 cohorts with $26 \%$ anteriorly-located left nerves in our previous study versus $18.6 \%$ in the present study may possibly be related to the inclusion of reoperative cases in the present study which had a slightly lower rate of anterior left vagus nerves $(14.5 \%)$, possibly due to previous dissection of the carotid sheath and shifting of the nerve due to scarring. The other source of discrepancy between our two studies is in the average size of the left lobe. In the present study, on the left, an anterior vagus nerve was significantly related to a lower lobe volume. The lobe volume for cases with an anteriorly-located vagus nerve was $13 \pm 19$ versus $18 \pm 24 \mathrm{~cm}^{3}$ for cases with a vagus nerve deep to or in-between the great vessels $(\mathrm{P}=0.036)$. In our previous study, the average left lobe volume was $13 \pm 18 \mathrm{~cm}^{3}$, so relatively smaller than the average left lobe volume in the present study. A large lobe may possibly displace the vagus nerve more posteriorly or laterally. This finding, however, contradicts the study by Ha et al. who found a very high rate of left anteriorly-located vagus nerves $(35 \%)$ in their cohort of patients undergoing thyroid US for evaluation before radiofrequency ablation. They found that the presence of a bulging nodule was a risk factor for a vulnerable nerve, that is, susceptible to be close to the radiofrequency probe tip, and therefore anterior or medial (4).

Finally, discrepancies among published studies may also be related to the subjective nature of the interpretation of
US images. We did not study the inter- or intra-operator consistency on repeated US exams describing the location of the vagus nerve, so that reports even from the same radiologist may have a certain amount of variability. The location of the vagus nerve actually seems to be a continuum from medial to medial-anterior then to lateral-anterior, lateral, lateral-posterior, etc. so that categorizing the exact location for reproducibility, even into 6 groups as we did, contributes to some variability and subjectivity.

\section{Conclusions}

The vagus nerve can be reliably located on US performed before thyroid surgery. The nerve was superficial to the great vessels on the left in $18.6 \%$ of cases and in $6.1 \%$ of cases on the right, confirming previous US studies. These findings may be useful for surgeons performing IONM and particularly continuous nerve monitoring, to preoperatively map the vagus nerves and avoid trauma to anteriorly-located nerves. Further studies are needed in normal and abnormal thyroids to better understand normal anatomy and the modifications that large lobes and nodules can induce in the anatomy of the vagus nerve and to elaborate a standardized description of the anatomic variations of the vagus nerve for US reporting.

\section{Acknowledgments}

Funding: None.

\section{Footnote}

Reporting Checklist: The authors have completed the STROBE reporting checklist. Available at https:// gs.amegroups.com/article/view/10.21037/gs-21-580/rc

Data Sharing Statement: Available at https://gs.amegroups. com/article/view/10.21037/gs-21-580/dss

Peer Review File: Available at https://gs.amegroups.com/ article/view/10.21037/gs-21-580/prf

Conflicts of Interest: All authors have completed the ICMJE uniform disclosure form (available at https://gs.amegroups. com/article/view/10.21037/gs-21-580/coif). DMH receives consulting fees from Medtronic ${ }^{\circledR}$ for consulting in the domain of neuromonitoring and intraoperative imaging. Medtronic ${ }^{\circledR}$ is a medical device company that 
commercializes a device for intraoperative neuromonitoring. This disclosure did not influence, however, the findings of the study or its' clinical implications. The other authors have no conflicts of interest to declare.

Ethical Statement: The authors are accountable for all aspects of the work in ensuring that questions related to the accuracy or integrity of any part of the work are appropriately investigated and resolved. The study was conducted in accordance with the Declaration of Helsinki (as revised in 2013). The study was approved by the institutional ethics board at Gustave Roussy and individual consent for this retrospective analysis was waived.

Open Access Statement: This is an Open Access article distributed in accordance with the Creative Commons Attribution-NonCommercial-NoDerivs 4.0 International License (CC BY-NC-ND 4.0), which permits the noncommercial replication and distribution of the article with the strict proviso that no changes or edits are made and the original work is properly cited (including links to both the formal publication through the relevant DOI and the license). See: https://creativecommons.org/licenses/by-nc-nd/4.0/.

\section{References}

1. Randolph GW, Dralle H, International Intraoperative Monitoring Study G, et al. Electrophysiologic recurrent laryngeal nerve monitoring during thyroid and parathyroid surgery: international standards guideline statement. Laryngoscope 2011;121 Suppl 1:S1-16.

2. Phelan E, Schneider R, Lorenz K, et al. Continuous vagal IONM prevents recurrent laryngeal nerve paralysis by revealing initial EMG changes of impending neuropraxic injury: a prospective, multicenter study. Laryngoscope 2014;124:1498-505.

3. Dionigi G, Chiang FY, Rausei S, et al. Surgical anatomy and neurophysiology of the vagus nerve (VN) for standardised intraoperative neuromonitoring (IONM) of the inferior laryngeal nerve (ILN) during thyroidectomy. Langenbecks Arch Surg 2010;395:893-9.

4. Ha EJ, Baek JH, Lee JH, et al. Clinical significance of vagus nerve variation in radiofrequency ablation of thyroid nodules. Eur Radiol 2011;21:2151-7.

5. Hartl DM, Bidault S, Girard E, et al. Ultrasound visualization of the vagus nerve for intraoperative neuromonitoring in thyroid surgery. Eur Radiol 2021;31:4063-70.
6. Walter U, Tsiberidou P, Kersten M, et al. Atrophy of the Vagus Nerve in Parkinson's Disease Revealed by HighResolution Ultrasonography. Front Neurol 2018;9:805.

7. Jang JH, Cho CS, Yang KS, et al. Pattern analysis of nerve enlargement using ultrasonography in chronic inflammatory demyelinating polyneuropathy. Clin Neurophysiol 2014;125:1893-9.

8. Lee JH, Cheng KL, Choi YJ, et al. High-resolution Imaging of Neural Anatomy and Pathology of the Neck. Korean J Radiol 2017;18:180-93.

9. Lamartina L, Bidault S, Hadoux J, et al. Can preoperative ultrasound predict extrathyroidal extension of differentiated thyroid cancer? Eur J Endocrinol 2021;185:13-22.

10. Clancy JA, Deuchars SA, Deuchars J. The wonders of the Wanderer. Exp Physiol 2013;98:38-45.

11. Gibson A. Bilateral Abnormal Relationship of the Vagus Nerve in its Cervical Portion. J Anat Physiol 1915;49:389-92.

12. Miyake N, Hayashi S, Kawase T, et al. Fetal anatomy of the human carotid sheath and structures in and around it. Anat Rec (Hoboken) 2010;293:438-45.

13. Schubert C, Grimm AS, Stahl JH, et al. Nerve ultrasound reference data in children from two to seven years. Clin Neurophysiol 2020;131:859-65.

14. Walter U, Tsiberidou P. Differential age-, gender-, and side-dependency of vagus, spinal accessory, and phrenic nerve calibers detected with precise ultrasonography measures. Muscle Nerve 2019;59:486-91.

15. Curcean AD, Rusu GM, Dudea SM. Ultrasound appearance of peripheral nerves in the neck: vagus, hypoglossal and greater auricular. Med Pharm Rep 2020;93:39-46.

16. Laucius $\mathrm{O}$, Balnyte R, Petrikonis $\mathrm{K}$, et al. Ultrasonography of the Vagus Nerve in the Diagnosis of Parkinson's Disease. Parkinsons Dis 2020;2020:2627471.

17. Holzapfel K, Naumann M. Ultrasound Detection of Vagus Nerve Atrophy in Bulbar Amyotrophic Lateral Sclerosis. J Neuroimaging 2020;30:762-5.

18. Niu J, Zhang L, Ding Q, et al. Vagus Nerve Ultrasound in Chronic Inflammatory Demyelinating Polyradiculoneuropathy and Charcot-Marie-Tooth Disease Type 1A. J Neuroimaging 2020;30:910-6.

19. Crossley JR, Aminpour N, Giurintano JP, et al. Neurosarcoidosis Directly Involving the Cervical Vagus Nerve. Ann Otol Rhinol Laryngol 2021;130:215-8.

20. Ben-Menachem E. Vagus-nerve stimulation for the treatment of epilepsy. Lancet Neurol 2002;1:477-82. 
21. Ottaviani MM, Wright L, Dawood T, et al. In vivo recordings from the human vagus nerve using ultrasoundguided microneurography. J Physiol 2020;598:3569-76.

22. Kandil E, Mohsin K, Murcy MA, et al. Continuous vagal monitoring value in prevention of vocal cord paralysis following thyroid surgery. Laryngoscope 2018;128:2429-32.

23. Lombardi CP, De Waure C, Mariani M, et al. Efficacy of continuous neuromonitoring in thyroid surgery: preliminary report of a single-center experience. Gland Surg 2019;8:336-42.

24. Onoda N, Noda S, Tauchi Y, et al. Continuous intraoperative neuromonitoring for thyroid cancer surgery: A prospective study. Laryngoscope Investig Otolaryngol 2019;4:455-9.

25. Stankovic P, Wittlinger J, Georgiew R, et al. Continuous intraoperative neuromonitoring (cIONM) in head and neck surgery-a review. HNO 2020;68:86-92.

26. Schneider R, Machens A, Lorenz K, et al. Intraoperative nerve monitoring in thyroid surgery-shifting current paradigms. Gland Surg 2020;9:S120-8.

27. Sedlmaier A, Steinmuller T, Hermanns M, et al. Continuous versus intermittent intraoperative neuromonitoring in complex benign thyroid surgery: A retrospective analysis and prospective follow-up. Clin

Cite this article as: Bidault S, Girard E, Attard M, Garcia G, Guerlain J, Breuskin I, Baudin E, Hadoux J, Garcia C, Lamartina L, Hartl DM. Preoperative ultrasound mapping of the vagus nerve in thyroid surgery. Gland Surg 2022;11(1):91-99. doi: $10.21037 / g s-21-580$
Otolaryngol 2019;44:1071-9.

28. Giovagnorio F, Martinoli C. Sonography of the cervical vagus nerve: normal appearance and abnormal findings. AJR Am J Roentgenol 2001;176:745-9.

29. Knappertz VA, Tegeler CH, Hardin SJ, et al. Vagus nerve imaging with ultrasound: anatomic and in vivo validation. Otolaryngol Head Neck Surg 1998;118:82-5.

30. Chen HH, Chen TC, Yang TL, et al. Transcutaneous Sonography for Detection of the Cervical Vagus Nerve. Ear Nose Throat J 2021;100:155-9.

31. Park JK, Jeong SY, Lee JH, et al. Variations in the course of the cervical vagus nerve on thyroid ultrasonography. AJNR Am J Neuroradiol 2011;32:1178-81.

32. Dionigi G, Kim HY, Wu CW, et al. Neuromonitoring in endoscopic and robotic thyroidectomy. Updates Surg 2017;69:171-9.

33. Ji YB, Ko SH, Song CM, et al. Feasibility and efficacy of intraoperative neural monitoring in remote access robotic and endoscopic thyroidectomy. Oral Oncol 2020;103:104617.

34. Papini E, Monpeyssen H, Frasoldati A, et al. 2020 European Thyroid Association Clinical Practice Guideline for the Use of Image-Guided Ablation in Benign Thyroid Nodules. Eur Thyroid J 2020;9:172-85. 